Received: 04.12 .2017

Revised: 20.03.2018

Accepted: 06.04.2018

DOI: $10.17804 / 2410-9908.2018 .2 .083-091$

\title{
A VARIATIONAL PRINCIPLE FOR THE DISTURBANCES OF PARTICLE VELOCITIES IN THE FLOW OF A PERFECTLY PLASTIC STRIP UNDER ROLLING
}

\author{
V. D. Solovei \\ Institute of Engineering Science, Ural Branch of the Russian Academy of Sciences, \\ 34 Komsomolskaya St., Ekaterinburg, Russian Federation \\ http://orcid.org/0000-0003-4696-8706 soloveivd@yandex.ru \\ Corresponding author. E-mail: soloveivd@yandex.ru \\ Address for correspondence: ul. Komsomolskaya, 34, 620049, Ekaterinburg, Russian Federation \\ Tel.: +7 (343) 37535 92; fax: +7 (343) 3745330
}

The slow plane flow of a perfectly plastic strip under rolling is considered. The Prandtl friction law is assumed to be valid on the contact surface between the strip and the roller. A variational principle enabling one to build a set of velocity disturbances in order to study the stability of the stationary flow of a perfectly plastic strip at an arbitrary time is proved.

Keywords: perfectly plastic strip rolling, stationary flow, disturbances of particle velocities in a stationary flow, variational principle.

\section{References}

1. Gorelik S.S. Rekristallizatsiya metallov i splavov [Recrystallizayion of metals and alloys]. Moscow, Metallurgiya Publ., 1978, 568 p. (In Russian).

2. Shtremel M.A. In: Prochnost splavov. Ch. II. Deformatsiya [Srength of alloys. Part II. Deformation]. Moscow, MISIS Publ., 1997, 526 p. (In Russian).

3. Gubernatorov V.V., Sokolov B.K., Gervasieva I.V., Vladimirov L.R. On the Formation of Band Structures in Structurally Homogeneous Materials upon Deformation. Fizicheskaya Mezomekhanika, 1999, vol. 2, nos. 1-2, pp. 157-162. (In Russian).

4. Gubernatorov V.V., Sycheva T.S., Vladimirov L.R., Matveeva V.S., Pyatygin A.I., Melnikov M.B. The corrugation phenomenon and formation of strained and recrystallized structure and texture in metallic materials. Part II. Cubic syngony. Fizicheskaya Mezomekhanika, 2002, vol. 5, no. 6, pp. 95-99. (In Russian).

5. Glansdorff P., Prigogine I. Thermodynamic Theory of Structure, Stability, and Fluctuations. London, Wiley, 1971.

6. Solovei V.D., Trukhin V.B. The stability of upsetting of a viscoplastic parallelepiped without friction. Deformatsiya i Razrushenie Materialov, 2014, no. 2, pp. 9-13. (In Russian).

7. Lamb, Sir Horace. Hydrodynamics, 6th ed., Cambridge Univ. Press, London, 1932. (Reprinted by Dover Pubs., New York, 1945).

8. Il'yushin A.A. Deformation of viscous-plastic body. Uch. zapiski MGU, Mekhanika, 1940, no. 39, pp. 3-81. (In Russian).

9. Freudenthal A.M., Geiringer H. The Mathematical Theories of the Inelastic Continuum. In: Flügge S., ed. Elasticity and Plasticity (Elastizität und Plastizität): Handbuch der Physik (Encyclopedia of Physics), vol 3 (6), Springer, Berlin, Heidelberg 1958.

10. Kolmogorov V.L. Mekhanika obrabotki metallov davleniem [Mechanics of metal forming]. Moscow, Metallurgia Publ., 1986, 688 p. (In Russian). 
11. Alekseev A.E. Nonlinear laws of dry friction in contact problems for linear theory of elasticity. Prikladnaya Mekhanika i Tekhnicheskaya Fizika, 2002, vol. 43, no. 4. pp. 16-169. (In Russian). 12. Solovei V.D. Possible velocity disturbances in the lag zone in the rolling of a perfectly plastic strip. Deformatsiya i Razrushenie Materialov, 2014, no. 12, pp. 23-26. (In Russian). 
Подана в журнал: 04.12.2017

УДК 539.3

DOI: $10.17804 / 2410-9908.2018 .2 .083-091$

\title{
ВАРИАЦИОННЫЙ ПРИНЦИП ДЛЯ ВОЗМУЩЕНИЙ СКОРОСТЕЙ ПЕРЕМЕЩЕНИЙ ИДЕАЛЬНО ПЛАСТИЧЕСКОЙ ПОЛОСЫ ПРИ ПРОКАТКЕ
}

\author{
В. Д. Соловей \\ Федеральное государственное бюджетное учреждение науки \\ Институт машиноведения Уральского отделения Российской академии наук, \\ ул. Комсомольская, 34, Екатеринбург, Российская Федерациия

 \\ Ответственный автор. Электронная почта: soloveivd@yandex.ru \\ Адрес для переписки: 620049, Екатеринбург, ул. Комсомольская, 34, Российская Федерация \\ Тел.: +7 (343) 375-35-92; факс: +7 (343) 374-53-30
}

Рассматривается медленное плоское течение идеально пластической полосы при прокатке. Трение на контакте полосы с валками определяется законом Прандтля. Доказывается вариационный принцип, который позволяет строить множество возмущений скоростей перемещений для исследования на устойчивость стационарного течения идеально пластической полосы в произвольный момент времени.

Ключевые слова: прокатка идеально пластической полосы, стационарное течение, возмущения стационарного течения, вариационный принцип.

\section{1. Введение}

Формирование периодических мезоструктур в металлах при прокатке [1] связано с потерей устойчивости деформируемых полос [2-4]. Исследование деформации твердого тела на устойчивость производится по отношению к некоторому множеству возмущений скоростей перемещений, удовлетворяющих дифференциальным уравнениям, вытекающим из законов сохранения, уравнениям состояния и граничным условиям [5]. Прокатываемая полоса имеет сложную форму области течения, что усложняет решение упомянутой нестационарной краевой задачи для возмущений скоростей перемещений.

В настоящей работе доказывается Вариационный принцип для возмущений скоростей перемещений медленного стационарного течения идеально пластической полосы при прокатке в произвольный момент времени, для которого возмущения ускорений заданы. Использование этого принципа позволяет при определении возмущений скоростей перемещений в произвольный момент времени заменить операции, связанные с интегрированием дифференциальных уравнений, относительно более простой операцией минимизации найденного функционала. Устойчивость течения полосы по отношению к найденным таким образом возмущениям скоростей перемещений в произвольный момент времени может быть исследована с помощью критерия локальной устойчивости в интегральном смысле, предложенного в работе [6].

\section{2. Стационарное течение идеально пластической полосы при прокатке}

Будем рассматривать плоское медленное течение идеально пластической полосы, для которого уравнения движения имеют следующий вид [7, 5]: 


$$
\rho v_{i, t}=\sigma_{i j, j}
$$

Здесь $v_{i}$ - компоненты вектора скорости перемещения частицы; $\sigma_{i j}-$ компоненты тензора напряжений; $\rho$ - плотность тела $(\rho=c o n s t)$; тензорные индексы принимают значения прямоугольных декартовых координат $x, y$; по дважды повторяющимся тензорным индексам $i, j, k, l$ происходит суммирование по всем возможным их значениям; запятая перед индексом обозначает частную производную по соответствующей координате или по времени.

Система уравнений для стационарного течения идеально пластического тела имеет вид [8-10]:

$$
\begin{gathered}
\sigma_{i j, j}=0 ; \\
v_{i, i}=0 ; \\
s_{i j}=2 \tau_{s} \varepsilon_{i j} / H ; \\
\varepsilon_{i j}=\left(v_{i, j}+v_{j, i}\right) / 2 ; \\
s_{i j}=\sigma_{i j}-\sigma \delta_{i j},
\end{gathered}
$$

где $\varepsilon_{i j}$ и $S_{i j}$ - компоненты тензоров скоростей деформаций и девиатора напряжений соответственно; $\sigma=\sigma_{i i} / 3$ - гидростатическое давление; $H=\sqrt{2 \varepsilon_{i j} \varepsilon_{i j}}-$ интенсивность скоростей деформации сдвига; $\tau_{s}$ - предел текучести на сдвиг; $\delta_{i j}-$ символ Кронекера. Напряжения на границе области течения полосы $p_{i}$ определяются формулой Коши:

$$
p_{i}=\sigma_{i j} n_{j}
$$

где $n_{j}$ - компоненты единичной внешней нормали к границе области течения. 


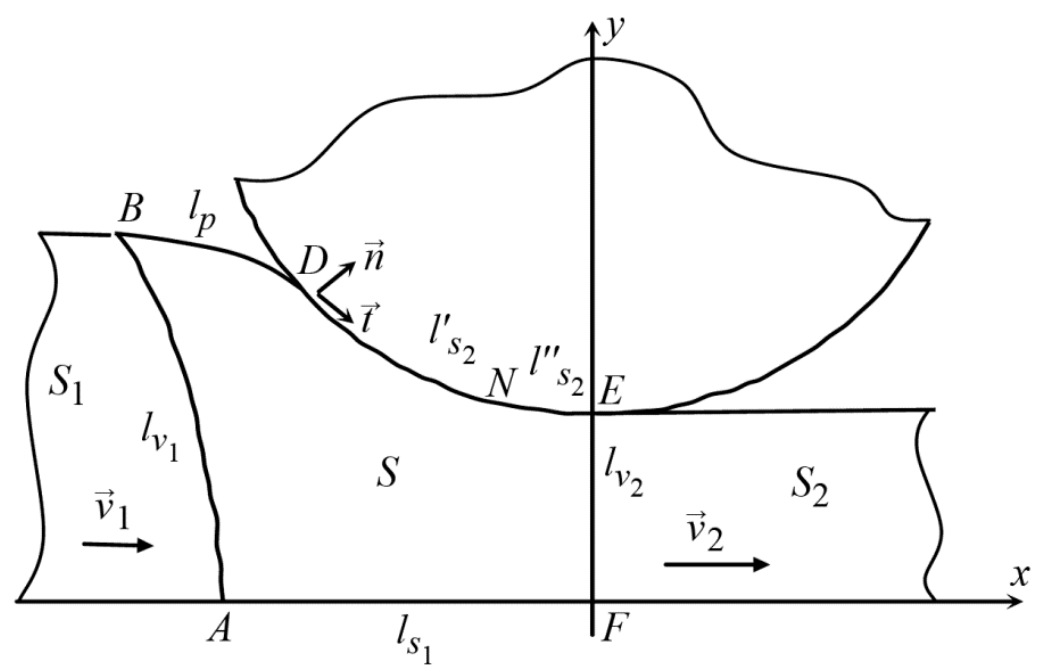

Схема прокатки полосы

На рисунке приведена схема процесса плоской прокатки полосы. Область $A B D E F(S)$ область течения полосы. На границе контакта полосы с валком $D E\left(l_{s 2}\right)$ имеет место проскальзывание частиц полосы по поверхности валка. В нейтральной точке $N$ касательные скорости перемещений частиц полосы и валка равны. Касательные составляющие скоростей перемещений частиц полосы $v_{t}$ в зоне отставания $D N\left(l_{s 2}^{\prime}\right)$ меньше, а в зоне опережения $N E\left(l_{s 2}^{\prime \prime}\right)$ - больше окружной скорости валка. Справа и слева от области течения изображены передний $\left(S_{2}\right)$ и задний $\left(S_{1}\right)$ жесткие концы полосы, движущиеся со скоростями $V_{2}$ и $V_{1}$ соответственно. Границы контакта области течения с жесткими концами полосы $S_{2}$ и $S_{1}$ обозначены, как $E F\left(l_{v 2}\right)$ и $A B\left(l_{v 1}\right)$ соответственно. Граница $B D\left(l_{p}\right)$ свободна от нагрузки, а граница $A F\left(l_{s 1}\right)$ представляет плоскость симметрии процесса прокатки. Индексы компонент векторов $t$ и $n$ относятся к локальной системе координат $\vec{t}, \vec{n}$.

Граничные условия для стационарного течения полосы имеют следующий вид:

$$
\begin{gathered}
p_{t}=\tau_{s} \text { на } l_{s 2}^{\prime}, \quad p_{t}=-\tau_{s} \text { на } l_{s 2}^{\prime \prime}, \quad v_{n}=0 \text { на } l_{s 2} ; \\
\left|p_{t}\right|=\tau_{s}, \quad v_{n}=v_{n}\left(V_{1}^{*}\right) \text { на } l_{v 1} ; \\
\left|p_{t}\right|=\tau_{s}, \quad v_{n}=V_{2}^{*} \text { на } l_{v 2} ; \\
p_{t}=0, \quad p_{n}=0 \text { на } l_{p} ; \\
p_{t}=0, \quad v_{n}=0 \text { на } l_{s 1} .
\end{gathered}
$$


Касательные напряжения трения $p_{t}$ на $l_{s 2}$ в (8) задаются приближенно с помощью закона трения Прандтля, который достаточно точно описывает закономерности внешнего трения в зонах больших нормальных напряжений [11]. Звездочкой обозначаются заданные величины. Полагаем, что решение системы уравнений (2)-(6) и граничных условий (8)-(12) известно.

\section{3. Возмущения стационарного течения идеально пластической полосы при прокатке}

Обозначим возмущение величины $f$ как $\tilde{f}$ и определим его следующим образом: $\tilde{f}=\delta f$, где $\delta$ - оператор изохронного, изокоординатного варьирования.

Возмущения величин относятся к произвольному моменту времени и являются функциями координат.

Система уравнений для возмущений стационарного течения идеально пластического тела имеет следующий вид:

$$
\begin{gathered}
\rho \tilde{v}_{i, t}^{*}=\tilde{\sigma}_{i j, j}, \\
\tilde{v}_{i, i}=0, \\
\tilde{s}_{i j}=2 \tau_{s} H^{-3}\left(-2 \varepsilon_{k l} \tilde{\varepsilon}_{k l} \varepsilon_{i j}+H^{2} \widetilde{\varepsilon}_{i j}\right), \\
\tilde{\varepsilon}_{i j}=\left(\tilde{v}_{i, j}+\tilde{v}_{j, i}\right) / 2 . \\
\tilde{s}_{i j}=\tilde{\sigma}_{i j}-\tilde{\sigma} \delta_{i j} .
\end{gathered}
$$

Уравнения (13)-(17) получаются варьированием уравнений (1), (3)-(6) с последующим удержанием только первых вариаций. Отметим, что возмущение ускорения $\tilde{v}_{i, t}^{*}$ в (13) задано.

Используя (15), можно убедиться, что мощность возмущения скорости деформации

$$
\tilde{W}=\tilde{s}_{i j} \widetilde{\varepsilon}_{i j} / 2
$$

является потенциалом для возмущения девиатора напряжения, то есть

$$
\tilde{s}_{i j}=\partial \tilde{W} / \partial \widetilde{\varepsilon}_{i j}
$$

Возмущения напряжений на границе тела определяются формулой

$$
\tilde{p}_{i}=\tilde{\sigma}_{i j} n_{j}
$$

Сформулируем граничные условия для возмущений переменных в произвольный момент времени. 
На границах $l_{s 2}, l_{v 1}, l_{v 2}$ и $l_{s 1}$ задаются возмущения скоростей перемещений

$$
\tilde{v}_{n}=0, \quad \tilde{v}_{t}=\tilde{v}_{t}^{*}
$$

На свободной границе $l_{p}$ задаются

$$
\tilde{v}_{n}=\tilde{v}_{n}^{*} \cdot \tilde{v}_{t}=\tilde{v}_{t}^{*} .
$$

При этом в зоне отставания $l_{s 2}^{\prime}$ волна возмущения $\tilde{v}_{t}^{*}$ задается двухпараметрической функцией [12]

$$
\tilde{v}_{t}^{*}=\sum_{k=1}^{P} \delta_{k} \sin \left[2 \pi k\left(l-l_{d}\right) /\left(l_{n}-l_{d}\right)\right]
$$

где $l$ - криволинейная координата на поверхности валка: $l=R \arcsin (x / R) ; l_{d}$ и $l_{n}-l_{- \text {ко- }}$ ординаты точек $D$ и $N$ соответственно; $\delta_{k}$ - малая амплитуда $k$-ой волны возмущения; параметры $\delta_{k}$ и $P$ произвольны. Остальные задаваемые на границе возмущения скоростей перемещений произвольны.

\section{4. Вариационный принцип для возмущений скоростей перемещений стационарного течения идеально пластической полосы}

Возмущения скоростей перемещений $\tilde{v}_{i}^{\prime}$, которые в произвольный момент времени удовлетворяют уравнению несжимаемости (14) и граничным условиям (21)-(23), будем называть кинематически возможными возмущениями скоростей перемещений. Величины со штрихом определяются как соответствующие им функции от кинематически возможных возмущений скоростей перемещений.

Покажем, что функционал

$$
\tilde{G}^{\prime}=\int_{S} \tilde{W}^{\prime} d S+\int_{S} \rho \tilde{v}_{i, t}^{*} \tilde{v}_{i}^{\prime} d S
$$

определенный на множестве кинематически возможных возмущений скоростей перемещений $\tilde{v}_{i}^{\prime}$, для действительных возмущений скорости перемещения $\tilde{v}_{i}$ принимает минимальное значение. Здесь $S$ - площадь области течения полосы.

Заметим, что возмущения ускорений $\tilde{v}_{i, t}^{*}$ в функционале (24) для рассматриваемого момента времени заданы.

Рассмотрим первую вариацию функционала $\delta G$, связанную с вариациями возмущений скоростей перемещений $\delta \tilde{v}_{i}$ относительно действительных возмущений скоростей перемещений $\tilde{v}_{i}$. Выражение (24), учитывая соотношения (19), (17), (14), (16) симметричность возмущения тензора напряжений $\tilde{\sigma}_{i j}$, используя теорему Гаусса-Остроградского, принимая во внимание (20), можно преобразовать к виду: 


$$
\delta \widetilde{G}=\int_{S}\left(-\tilde{\sigma}_{i j, j}+\rho \tilde{v}_{i, t}^{*}\right) \delta \tilde{v}_{i} d S+\oint_{l} \tilde{p}_{i} \delta \tilde{v}_{i} d l
$$

где второй интеграл берется по всей границе области течения.

Из (25), (13) и граничных условий $(21),(22)$ следует равенство

$$
\delta \widetilde{G}=0
$$

Необходимое условие минимума функционала $\widetilde{G}$ для действительных возмущений скоростей перемещений $\tilde{v}_{i}$ доказано.

Рассмотрим вторую вариацию функционала $\delta^{2} \tilde{G}$, связанную с вариациями возмущений скоростей перемещений $\delta \tilde{v}_{i}$ относительно действительных возмущений скоростей перемещений $\tilde{v}_{i}$.

Из (24) следует

$$
\delta^{2} \tilde{G}=\int_{S} \delta^{2} \tilde{W} d S
$$

Учитывая (19) и (15), можно написать выражение

$$
\delta^{2} \tilde{W}=4 \tau_{s} H^{-3}\left(\varepsilon_{i j} \varepsilon_{i j} \delta \widetilde{\varepsilon}_{k l} \delta \widetilde{\varepsilon}_{k l}-\varepsilon_{i j} \delta \widetilde{\varepsilon}_{i j} \varepsilon_{k l} \delta \widetilde{\varepsilon}_{k l}\right) .
$$

Выражение в скобках, согласно неравенству Коши-Буняковского, неотрицательно и равно нулю, если $\delta \widetilde{\varepsilon}_{i j}=c \varepsilon_{i j}$.

Из (27) и (28) следует неравенство (если исключить случаи, когда $\delta \widetilde{\varepsilon}_{i j}=c \varepsilon_{i j}$ )

$$
\delta^{2} \tilde{G}>0
$$

Достаточное условие минимума функционала $\widetilde{G}$ для действительных возмущений скоростей перемещений $\tilde{v}_{i}$ доказано.

Таким образом, функционал (24) для действительных возмущений скоростей перемещений $\tilde{v}_{i}$ принимает минимальное значение.

В заключение отметим, что в произвольный момент времени для каждого заданного поля возмущений ускорений $\tilde{v}_{i, t}^{*}$, минимизируя функционал (24), можно решить соответствующую краевую задачу для возмущений скоростей перемещений $\tilde{v}_{i}$. Для произвольного момента времени, варьируя возмущения ускорений $\tilde{v}_{i, t}^{*}$, можно получить искомое множество возмущений скоростей перемещений $\tilde{v}_{i}$ для исследования стационарного течения идеально пластической полосы при прокатке на устойчивость. 


\section{5. Заключение}

Найден функционал, имеющий минимум для действительных возмущений скоростей перемещений стационарного течения идеально пластической полосы при прокатке в произвольный момент времени. Вариационный принцип позволяет эффективно определять возмущения скоростей перемещений в произвольный момент времени, необходимые при исследовании устойчивости течения идеально пластической полосы при прокатке.

\section{Литература}

1. Горелик С. С. Рекристаллизация металлов и сплавов. - М. : Металлургия, 1978. - 568 с. 2. Штремель М. А. Прочность сплавов. - М . : Изд-во МИСИС, 1997. - 526 с. - Ч. II : Деформация.

3. О формировании полосовых структур в структурно-однородных материалах при деформации / В. В. Губернаторов, Б. К. Соколов, И. В. Гервасьева, Л. Р. Владимиров // Физическая мезомеханика. - 1999. - Т. 2, № 1-2. - С. 157-162.

4. Явление гофрирования и формирования структуры и текстуры в металлических материалах при деформации и рекристаллизации: 2. Сплавы кубической сингонии / В. В. Губернаторов, Т. С. Сычева, Л. Р. Владимиров, В. С. Матвеева, А. И. Пятыгин, М. Б. Мельников // Физическая мезомеханика. - 2002. - Т. 5, № 6. - С. 95-99.

5. Гленсдорф П., Пригожин И. Термодинамическая теория структуры, устойчивости и флуктуаций. - М. : Мир, 1973.

6. Соловей В. Д., Трухин В. Б. Устойчивость процесса осадки вязкопластического параллелепипеда без трения // Деформация и разрушение материалов. - 2014. - № 2. - С. 9-13.

7. Лэмб Г. Гидродинамика / пер. с 6-го англ. издания А. В. Гермогенова, В. А. Кудрявцева / под ред. Н. А. Слёзкина. - М. ; Л. : ОГИЗ «Гостехтеоретиздат», 1947. - 928 с.

8. Ильюшин А. А. Деформация вязко-пластичного тела // Уч. зап. МГУ. Механика. М. : Изд-во МГУ, 1940. - Вып. 39. - С. 3-81.

9. Фрейденталь А., Гейрингер Х. Математические теории неупругой сплошной среды. М. : ГИФМЛ, 1962.

10. Колмогоров В. Л. Механика обработки металлов давлением. - М. : Металлургия, 1986. 11. Алексеев А. Е. Нелинейные законы сухого трения в контактных задачах линейной теории упругости // Прикладная механика и техническая физика. - 2002. - Т. 43, № 4. C. 161-169.

12. Соловей В. Д. Возможные возмущения скоростей перемещений в зоне отставания при прокатке идеально пластической полосы // Деформация и разрушение материалов. - 2014. № 12. - С. 23-26. 